\title{
LAS IMPLICACIONES POLÍTICAS DEL ABSTENCIONISMO ELECTORAL EN EL ESTADO DE HIDALGO
}

Enrique López Rivera*

\section{RESUMEN}

En el número anterior de Xihmai, Pedro Liedo se cuestiona en su artículo sobre las implicaciones que pueden tener las democracias actuales con índices elevados de abstencionismo electoral. Dentro de su razonamiento, el aut

or se pregunta si es posible gobernar con una minoría cuando, en sentido teórico, la democracia se entiende como el gobierno de las mayorías. Este ensayo busca ampliar esta discusión ofreciendo algunos elementos de análisis para el caso del estado de Hidalgo.

\section{ABSTRACT}

In the previous volume of "Xihmai", Pedro Liedo explores the possible implications for democracies with high levels of voter absenteeism. The author questions whether it is possible to govern with a minority and still be called a democracy when, in theory, a democracy is understood to be government by the majority. This essay broadens the discussion by including some points of analysis pertinent to the State of Hidalgo.

\section{INTRODUCCIÓN}

¿Por qué es pertinente estudiar el abstencionismo electoral en Hidalgo? Encuentro al menos dos razones; la primera es que Hidalgo pasó de ser un estado participativo a otro en donde aumenta gradualmente la abstención. Bien vale la pena cuestionarse qué ocurrió con aquel entusiasmo electoral de antaño. La segunda razón es que Hidalgo presenta rasgos disímiles en las elecciones federales, locales y municipales. Es decir, el abstencionismo no se presenta con la misma magnitud ni dimensión en todos los procesos electorales.

En este sentido, las elecciones presidenciales y municipales siguen siendo las más concurrentes por los votantes hidalguenses mientras 
que los otros procesos no generan la misma participación. En el caso de los comicios para elegir a los integrantes del poder legislativo (diputados) los electores presentan la mayor apatía.

Este comportamiento disímil puede tener un mensaje implícito. Los electores parecen más involucrados en las elecciones de autoridades ejecutivas que legislativas. Tal parece que algunos rasgos del presidencialismo exacerbado que tuvimos en México todavía influyen en los electores, dando mayor importancia a los procesos electorales en donde se elige a un representante que preside un órgano ejecutorio, que a los cuerpos colegiados que realizan actividades legislativas.

* Licenciado en Ciencia Política, egresado de la Maestría en Estudios de Población en la UAEH, Maestro en Estudios Sociales por la UAM y becario del CONACYT en el programa de Doctorado en Estudios Sociales de la UAM. Catedrático de la Escuela de Ciencias Humanas de la Universidad La Salle Pachuca. uam_lore04@hotmail.com.

Una mirada a las tendencias de participación y abstencionismo en Hidalgo

La gráfica 1 muestra las últimas seis elecciones de gobernador en Hidalgo. El abstencionismo ha superado el $50 \%$ en los últimos tres procesos. En 1993 la abstención llegó a 59.4\%, en 1999 baja a 50.9\% y en 2005 vuelve a subir a $54.6 \% .1$ 
GRÁFICA 1 TENDENCIA DE LA PARTICIPACIÓN Y ABSTENCIÓN EN ELECCIONES PARA ELEGIR GOBERNADOR 1975

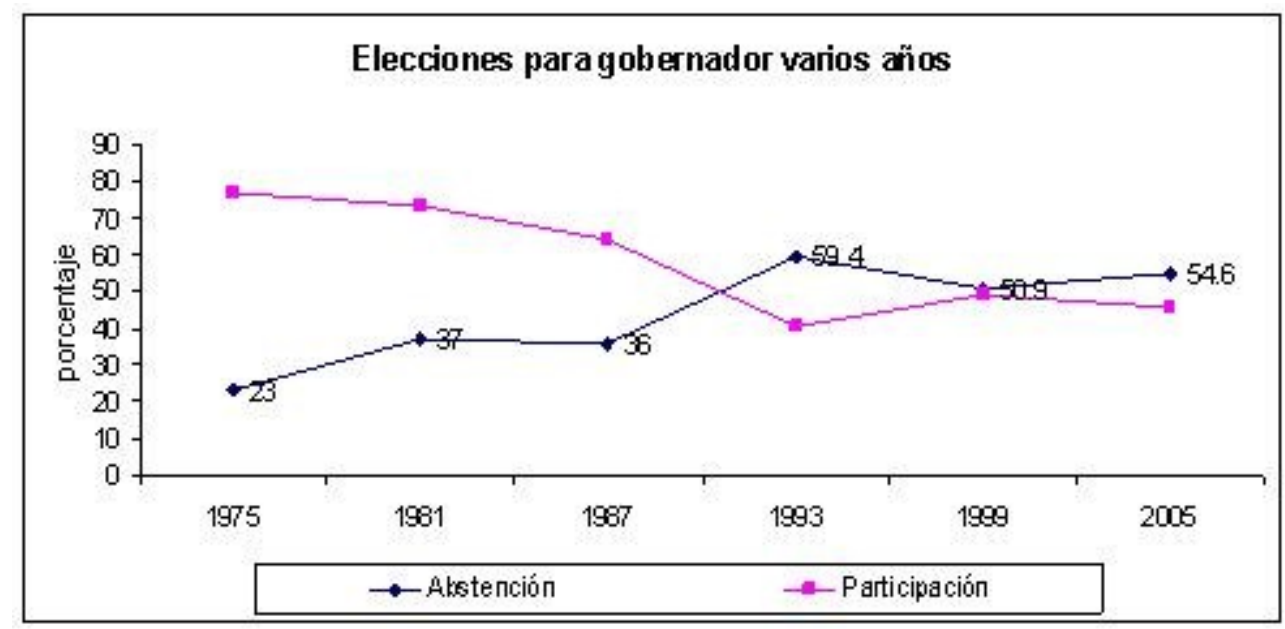

Elaboración propia con datos del IEE.

Los resultados marcan una tendencia decreciente de la participación electoral en los últimos años. No obstante, lo que resulta más revelador de analizar son los años previos a 1993. Aquí encontramos una participación en elecciones propia de países europeos. Por ejemplo, en 1975 el $77 \%$ de los electores votaron. En 1981 y 1987 más del 60\% acudieron a la urna.

Observando la gráfica anterior nos percatamos que entre 1987 y 1993 se invierten las líneas de participación y abstención electoral. Previo a 1993 Hidalgo era una entidad muy entusiasta. Después de 1993 Hidalgo se convierte en una entidad apática. ¿Qué ocurrió en esos años? parece ilógico que con mejores condiciones de competencia los ciudadanos opten por la apatía antes que por la participación. Un especialista considera al respecto que: 
...parece una gran contradicción a veces sin sentido, el hecho de que en sociectades en las cuales se han ampliado los derec hos al sufragio, se han mejorado las reglas de lacompetencia elec toral y existe una mayor in formación sobre los asuntos politicos, sobre los partidos, los candidatos y las campañas, el abstencionismo es creciente.'

Algunos es pedalistas han analizado esta incongruen da. Entre ellos, la explicación mäs recurrente consiste en la creciente insatisfación de los ciudadanos on la democracia. Sin embargo, los más criticos aluden a una serie de mecan ismos utilizados por el viejo régimen para manipular los datos electorales. En esta última posición, algunos autores, aseguran que sin la existencia de instituciones autónomas las elecciones podian manipularse en —al menosdos sentidos: no sólo se favorecia al partido en el gobierno aumentand o su votación sino que también se restaban votos a la oposición. Este mecanismo engrosaba por mucho el universo de votantes. Por tanto, la participadón casi siempre era muy elevada. Dicho de otra manera, ahora que existen instituciones que organizan, vigilan y dan certeza a los procesos electorales aparece el verdadero ros tro de los ciudadanos cada vez más a páticos y ausentes de las urnas

Pero esto es sólo una reducida parte del fenómeno que nos ocupa. Si obserwamos otros indicadores las caracteristicas parecen modificarse. Ya se anticipaba el ausentismo en elecciones de representantes de órganos legislativos que por mucho son las menos participativas como lo muestra la siguiente gräfica.

GRÁFICA TENDENCIAS DE PARTICIPACIÓN 2 ELECCIONES DE DIPUTADOS FEDERALES EN HIDALGO 1988 2003.

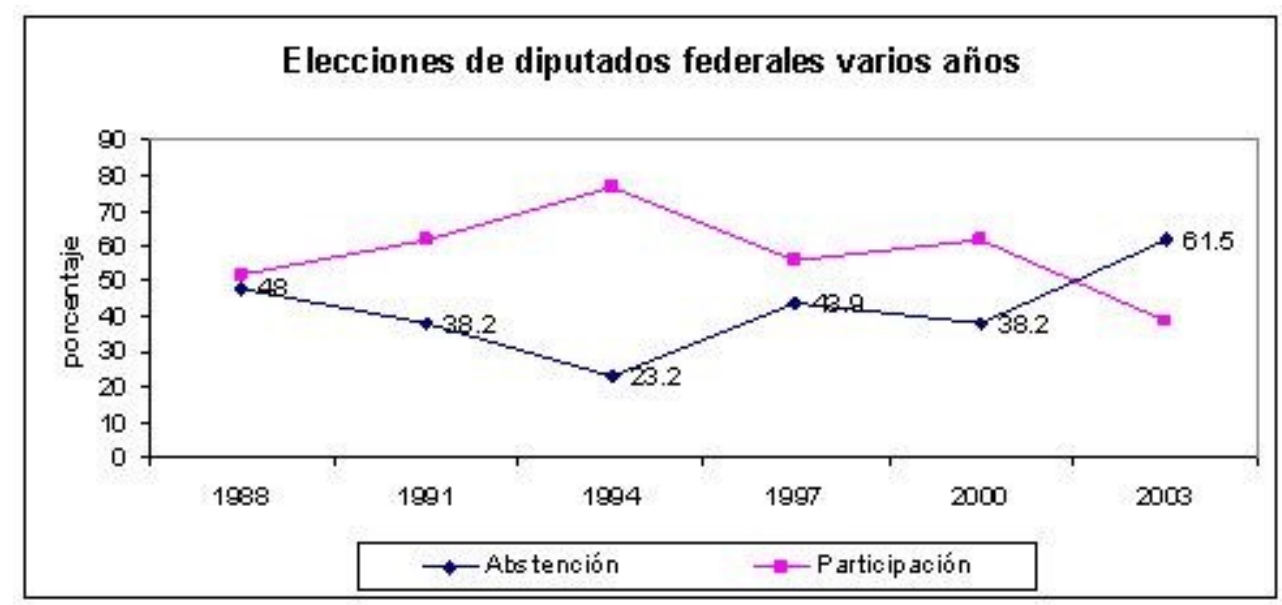

Elaboración propia con datos de Atlas Electoral Federal de México 1991-2003. IFE . 
La gráfica 2 muestra los resultados de las elecciones de diputados de mayoría relativa desde 1988 hasta 2003. En este recorrido se observa una tendencia creciente de la participación de 1991 a 1994 encontrando su máximo valor en este último año con una participación de $77 \%$ del padrón electoral.

A partir de 1994 la abstención comienza a ganar terreno. En sólo tres años se incrementa en 20 puntos porcentuales, baja un poco en 2000, y en 2003 se incrementa de manera considerable con $61.5 \%$ de ciudadanos que no ejercieron su voto.

Esto nos conduce a analizar otros elementos. El poder legislativo es, a los ojos de algunos ciudadanos, una institución que dificulta la gobernabilidad. Su naturaleza reciente multicolor y plural no ha permitido los consensos necesarios para llevar a cabo reformas fundamentales y, por ello, los votantes han mostrado cierto rechazo a la hora de elegirlos a través del voto. Para reforzar lo anterior basta con observar que las elecciones en donde sólo se convoca a renovar el poder legislativo, el ausentismo es mayor. Mientras que en elecciones legislativas que coinciden con presidenciales el porcentaje de votación es mayor.

Sin duda las elecciones que más entusiasmo generan entre los hidalguenses son las presidenciales ya que la elección presidencial es la más publicitada, la que genera más interés y mayor involucramiento de los medios masivos. Sin embargo, en 1988 ocurrió algo peculiar. En aquella ocasión la competencia política fue histórica, la campaña levantó muchas expectativas, los contendientes visitaron varias veces el estado y, sin embargo, el $48 \%$ decidió no votar, como se observa en la siguiente gráfica 
GRÁFICA

TENDENCIAS

ELECCIONES

DE PARTICIPACIÓN

PRESIDENCIALES

Y

1976

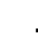

EN

ABSTENCIÓN

HIDALGO

$2000^{*}$

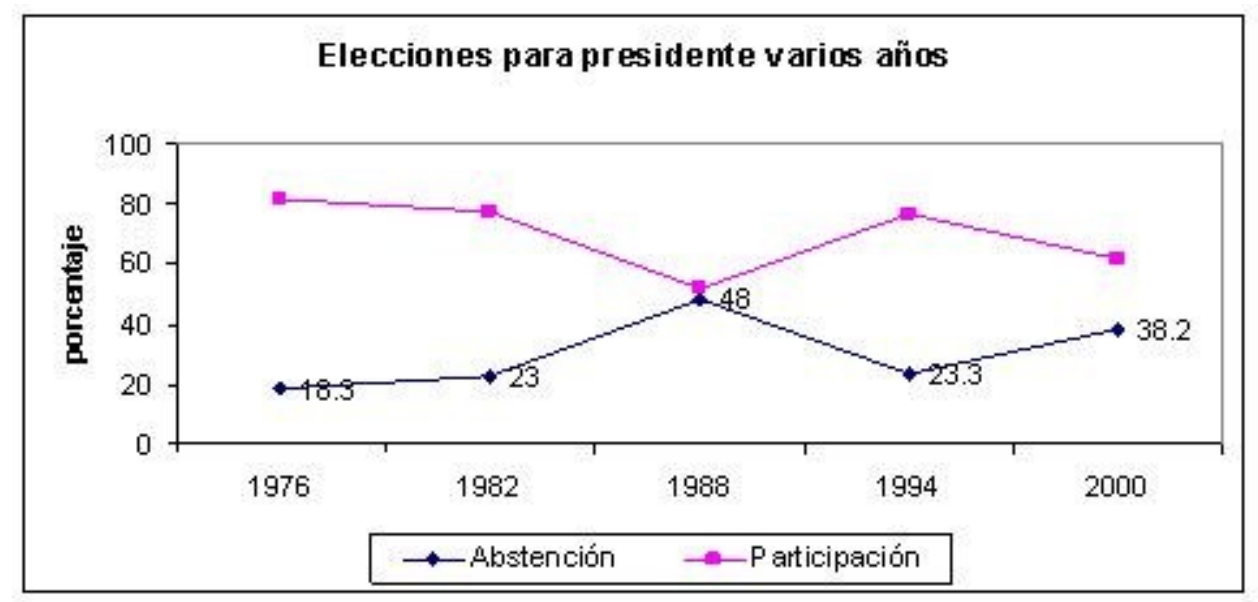

Elaboración propia con datos de Atlas Electoral Federal de México 1991 - 2003, IFE.

* No se incluye la elección de 2006 puesto que, a la entrega del artículo, el Tribunal Electoral no no había resuelto si anularía algunas casillas impugnadas.

En 1988 la oposición ganó importantes espacios de poder en el estado. Lo cual nos lleva a cuestionarnos lo siguiente: ¿la elevada participación electoral facilita o dificulta la alternancia en el poder? Regresaremos más adelante con esta pregunta.

La gráfica anterior también nos muestra que en 1976 casi el $90 \%$ de los ciudadanos sufragó; en 1982 y 1994 el 77\%, en 2000 el 62\% mientras que en 1988 sólo el 52\% votó, siendo este porcentaje el menor del pasado reciente en el estado. Es decir, en 1988 la abstención alcanzó su máximo valor con $48 \%$.

En el ámbito municipal el comportamiento de las dos variables, abstención y participación, presenta cambios significativos. En este tipo de elección se pueden observar al menos dos tendencias. La primera de gran variabilidad y la segunda de cierta constancia. 
GRÁFICA

TENDENCIAS DE PARTICIPACIÓN Y ABSTENCIÓN ELECCIONES PARA RENOVAR AYUNTAMIENTOS EN HIDALGO 1987

2005.

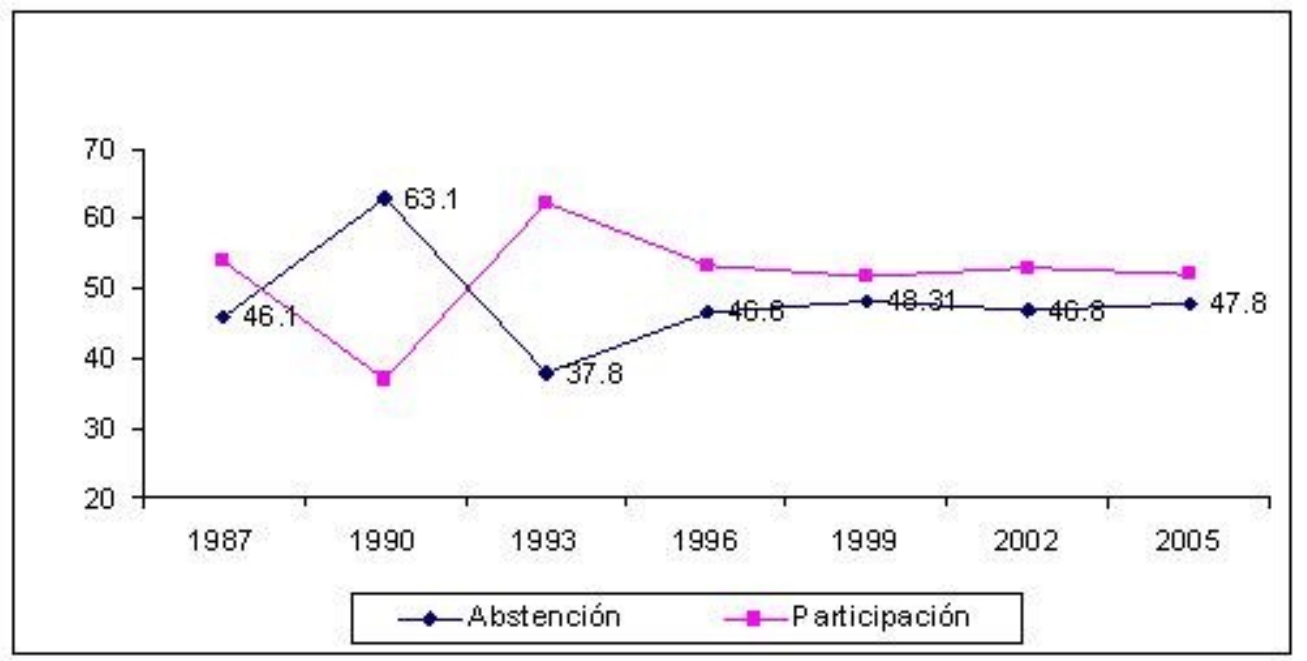

Elaboración propia con datos del IEE.

En la gráfica anterior se puede observar que entre 1987 y 1993 el abstencionismo fue muy variable. A partir de 1996 ha permanecido constante con niveles cercanos al $50 \%$. Las elecciones municipales tienen su propia lógica. Aquí las autoridades regularmente son más cercanas a los ciudadanos ya que los candidatos radican en el municipio correspondiente y, por tanto, están involucrados con los problemas cotidianos. Los grupos de poder ejercen mayor influencia en este tipo de elección y los procesos de alternancia en el estado se han dado justamente desde el ámbito municipal, no obstante, desde 1996 casi el $50 \%$ de los ciudadanos no participa en estas elecciones. 
Algunos elementos adicionales del abstencionismo

Derivado del diagnóstico anterior podemos resaltar algunas características propias del elector hidalguense. La primera enseñanza es que el abstencionismo se presenta con distinto rostro en cada elección. Dicho de otra manera, hay elecciones que estimulan una participación electoral copiosa y, otras en cambio, se encuentran lejanas del interés del votante. Pero no sólo eso, hay suficientes ejemplos de comicios que con baja participación electoral han facilitado la alternancia en el poder, o bien, comicios que tienen alta participación electoral y ratifican al partido en el gobierno. Lo cierto es que el abstencionismo es una más de las variables que acompañan al resultado de una elección. Por ello, la abstención no debe ser una preocupación para conformar gobiernos elegidos por los ciudadanos. No lo es, dado que la convocatoria es amplia, acuden a votar los que así lo deciden. Aquellos que no votan no descalifican de tajo al sistema democrático. Pueden permanecer ajenos a la política y, por eso no se les debe de juzgar. Ahí radica la explicación de que la democracia no es un sistema de gobierno de mayorías sino uno de élites dispuestas a participar en la conformación de gobiernos a través del voto. En suma, el sistema democrático puede sobrevivir con mayorías y minorías dispuestas a participar o abstenerse.

Sin embargo, existe una gran preocupación por los gobiernos elegidos con reducida participación electoral. Algunos autores han sugerido la obligatoriedad del sufragio como ya ocurre en algunos países de América Latina. Sin embargo, obligar al ciudadano a participar en elecciones no es el mecanismo más adecuado para generar una cultura cívica democrática. Las autoridades electorales, por su parte, realizan continuamente foros de discusión y análisis sobre el impacto que tiene el abstencionismo en las democracias actuales. Gracias a estos espacios hoy se tiene un buen bagaje teórico sobre el tema. Pero esto no parece ser suficiente. Son muchas las voces que se pronuncian por erradicar de tajo al abstencionista. Aquellos que consideran un peligro los porcentajes de ausentismo y que ven en un ciudadano que no vota a un enemigo del sistema, a un inconforme con la democracia, a un apático de la cuestión pública. 
¿En donde radica la preocupación por el elevado abstencionismo? ¿A quién afecta la reducida participación en elecciones? ¿Por qué se rechaza al ciudadano que no vota?

Las implicaciones políticas de la abstención electoral

En el escenario político actual de mayor competencia electoral, pluralismo en casi todos los órganos de representación e instituciones autónomas; el hecho de que los ciudadanos sean cada vez menos participativos en elecciones no parecería ser un riesgo para nadie. Sin embargo, algunos sectores hacen lo posible para que los ciudadanos acudan a sufragar sin falta. La intensión no es del todo mala. Pero a mi juicio parece mal intencionada. Me explico. Los gobiernos elegidos por una cantidad numerosa de votos llevan consigo una aceptación generalizada, una voluntad general como decía Rousseau, una alta dosis de legitimidad como aseguran los politólogos. Eso es lo que se busca; gobiernos con mucha aceptación, con legitimidad electoral, con alto respaldo popular. Por eso, políticos, autoridades y algunos sectores incentivan la participación en elecciones. Pensando que un gobierno será incuestionable y tendrá altos márgenes de gobernabilidad si es elegido por una mayoría. Pero eso no es del todo cierto. La legitimidad se puede adquirir por otros medios no sólo a través de elecciones. Un teórico norteamericano asegura que la aceptación de un gobierno se puede lograr a partir de la eficacia en sus decisiones, de su transparencia en el ejercicio del poder y en su capacidad de generar acuerdos. 3

Ahora bien, el ciudadano que no vota tampoco está exento de todo mal. La decisión de no votar es una decisión individual que tiene repercusiones sociales. El abstencionista pierde la oportunidad de castigar o recompensar con su voto a partidos políticos o representantes que hicieron bien su trabajo o aquellos que deben ser removidos del cargo. 
Por último, es necesario dejar claro que las elecciones son mecanismos indispensables en una democracia; sin embargo, existen otros puentes para poder participar, incidir o involucrarse en política. Los ciudadanos tienen múltiples alternativas para formar parte de la res pública. Entre ellas se encuentran la participación en organizaciones civiles, sindicatos, asociaciones vecinales, por citar algunas. Lo cierto es que la participación electoral es la expresión mínima de participación política que poco a poco parece perder terreno en el ánimo del ciudadano.

\section{NOTAS}

1 Vale la pena mencionar que antes de 1993 las elecciones eran reguladas por la Comisión Federal Electoral Delegación Hidalgo los resultados electorales, por tanto, no cuentan con la validez de una institución autónoma. En 1995 se crea el Instituto Estatal Electoral de Hidalgo organismo ciudadanizado y autónomo que organiza las elecciones locales. Una recopilación sobre las instituciones que han organizado las elecciones en el estado puede verse en, Alcalá Montaño, Alfredo. "Hidalgo entre el autoritarismo y la democracia. El Instituto Estatal Electoral y la alternancia municipal en el estado 19952002" en Revista Mexicana de Estudios Electorales No. 4, Julio diciembre

2004.

2 Bahena Álvarez, Fernando. "Participación y abstencionismo en el estado de México: elementos para su estudio", en Arreola Ayala, Álvaro (coord.) Comportamiento político y tendencias electorales en el estado de México, Ed. IEEM, México 2003, p.127.

3 Deutsch Karl. Política y gobierno, Ed. FCE, México 1998, p. 26. 


\section{REFERENCIAS}

ALCALÁ, Alfredo. Hidalgo entre el autoritarismo y la democracia. El Instituto Estatal Electoral y la alternancia municipal en el estado 19952002 en Revista Mexicana de Estudios Electorales, No. 4, julio diciembre 2004.

ALCUBILLA, Enrique. Abstencionismo, en Diccionario Electoral, Instituto Interamericano de Derechos Humanos (IIDH) Centro Interamericano de Asesoría y Promoción Electoral (CAPEL), San José de Costa Rica, 2000

BAHENA, Fernando. Participación y abstencionismo en el estado de México: elementos para su estudio, en Arreola Ayala, Álvaro (coord.) Comportamiento político y tendencias electorales en el estado de México, Ed. IEEM, México 2003.

DEUTSCH, Karl. "Política y gobierno", Ed. FCE, México 1998.

NOHLEN, Dieter. "Ampliación de la participación política y reducción del abstencionismo: ejes de una cultura democrática y una nueva ciudadanía para el siglo XXI", Conferencia Protocolo de Tikal, San José de Costa Rica, Septiembre 2003.

---“La participación electoral como objeto de estudio", en Elecciones, Oficina Nacional de Procesos Electorales (ONPE) Año 3, No. 2, diciembre de 2004. 